Cahiers de philosophie de l'université de

\title{
Pavel Florensky : une philosophie antinomique de la culture et de l'individualité
}

\section{Vladimir Porus}

\section{(2) OpenEdition}

1 Journals

Édition électronique

URL : https://journals.openedition.org/cpuc/967

DOI : $10.4000 /$ cpuc. 967

ISSN : 2677-6529

Éditeur

Presses universitaires de Caen

\section{Édition imprimée}

Date de publication : 31 octobre 2011

Pagination : 75-92

ISBN : 978-2-84133-385-1

ISSN : 1282-6545

\section{Référence électronique}

Vladimir Porus, «Pavel Florensky : une philosophie antinomique de la culture et de l'individualité », Cahiers de philosophie de l'université de Caen [En ligne], 48 | 2011, mis en ligne le 01 septembre 2020, consulté le 01 février 2023. URL : http://journals.openedition.org/cpuc/967 ; DOI : https://doi.org/ 10.4000/cpuc.967

\section{(c) (†) 8}

Creative Commons - Attribution - Pas d'Utilisation Commerciale 4.0 International - CC BY-NC 4.0 https://creativecommons.org/licenses/by-nc/4.0/ 


\section{Pavel Florensky: \\ une philosophie antinomique de la culture et de l'individualité ${ }^{1}$}

\section{$\mathrm{L}$}

A CONTRADICTION INTERnE de la culture européenne est celle qui existe entre la singularité de l'existence et la totalité de ce qu'on peut appeler les «universaux culturels». Cette contradiction reproduit d'une façon formelle la structure du rapport entre la volonté libre de l'individu et la volonté divine (on peut rappeler le débat classique entre Luther et Érasme au $\mathrm{XVI}^{\mathrm{e}}$ siècle). Il ne s'agit que d'une reproduction formelle parce que durant la "sécularisation » de la culture européenne ses « universaux» ont été « séparés » de leur fondement transcendant. La contradiction « descend» des sommets théo-métaphysiques jusqu'au fond des conflits entre l'individualité humaine libre et la contrainte des matrices qui «impriment» sur «le matériau humain» les formes standardisées de sa «sociabilité» (tous les philosophes de Feuerbach et Marx à Sartre et Heidegger, Durkheim et Lévi-Strauss s'accordent sur ce point).

Dans sa philosophie, Vladimir Soloviev (1853-1900) donne une valeur théologique et métaphysique à cette contradiction. Il la décrit comme une conséquence de la catastrophe théo-cosmique. L'opposition entre l'individuel et le total dans la vie humaine est représentée dans cette philosophie comme un état morbide et dénaturé, c'est-à-dire comme une perversion de l'être qui perd son authenticité. La catastrophe a aussi divisé le monde intérieur de l'homme dans lequel l'existence individuelle et égoïste entre en conflit avec l'essence divine. Mais Soloviev croit que le dépassement de la division est, en fin de compte, une négation de l'individualité pour la victoire sur le mal et la souffrance, une libération de l'homme de la liberté qui l'asservit.

1. Cet article a été écrit dans le cadre du projet franco-russe "Problème du "Moi": traditions et actualités» soutenu par le Centre de recherches fondamentales de l'École des hautes études en sciences économiques, université d'État (Moscou) (Programme de 2011). 
Le paradoxe qui représente la condition de la liberté comme un refus de celle-ci est illusoire: Soloviev utilise le même mot pour deux entités différentes. La première est une liberté irrationnelle, un arbitraire à l'aide duquel l'existence coupable et souffrante essaye de compenser son défaut: sa volonté pécheresse lance un défi à Dieu. La seconde est une liberté véritable coïncidant avec la nécessité morale, c'est une liberté de la raison qui assume la responsabilité morale. La seconde liberté doit vaincre dans ce conflit. C'est une condition pour rétablir l'omni-unité.

La philosophie de l'omni-unité résout-elle la contradiction de la culture? Oui, si l'on donne un fondement religieux aux universaux culturels. Non, si la culture est autonome par rapport à la religion. La question de la culture européenne se transforme ainsi en question sur son rapport au christianisme.

Cette question devient un objet central de réflexion sur la culture pendant les $\mathrm{XIX}^{\mathrm{e}}$ et $\mathrm{XX}^{\mathrm{e}}$ siècles en Russie. La réalité en effet met en question la capacité du christianisme à résister à la crise culturelle totale et aux cataclysmes postérieurs politiques, économiques et militaires. Dans ses derniers travaux Soloviev exprime d'une manière précise un pressentiment de la crise universelle prochaine. Dans les images apocalyptiques du philosophe on voit une reconnaissance de l'échec du christianisme en tant que projet culturel.

Le mouvement «réformiste» dans la philosophie russe religieuse (Nicolas Berdiaev, Léon Chestov, Dmitri Merejkovski, etc.) est inspiré par l'idée de redonner un rôle créateur à la culture du christianisme, de lui rendre un leadership spirituel perdu. La cause de la perte, comme le croit Berdiaev, est la suivante: l'église chrétienne a négligé un potentiel créatif de l'esprit humain et a remplacé le soin de le développer par un suivi formel des règles et des prescriptions de la vie religieuse. Résultat, le christianisme perd son âme, il se transforme en une institution dont les fonctions sont de soutenir la stabilité illusoire de l'ordre universel (ainsi que social) qui est déjà privé de sa justification spirituelle.

Dans le processus cosmique et universel du développement l'homme dépasse une stabilité relative de la vie physique et matérielle qui semblait absolument stable. Il comprend que cette dimension physique de la vie n'est pas l'être mais une routine. La crise pénible au cours de laquelle on passe des symboles et des phénomènes matériels et physiques à la révélation de la vie spirituelle supérieure embrasse tous les domaines. C'est une crise universelle.

Mais l'Église n'est pas prête à résister à cette crise. Elle est «contre toute recherche de la vie spirituelle supérieure, elle retient l'homme au niveau 
inférieur et enfantin $»^{2}$. Il est nécessaire de renouveler la conscience religieuse et, par conséquent, l'Église tout entière.

Le chemin des «réformateurs» exige de changer le rapport entre la spiritualité individuelle et les universaux culturels. La contradiction entre l'individu et la culture est transférée à l'intérieur de l'individu où elle devient la cause de la tragédie spirituelle et élève chez lui la possibilité de la catharsis, à condition de se débarrasser du mal d'une façon véritable (et non d'une façon formelle et illusoire). L'homme doit assimiler la catastrophe universelle pour la dépasser à l'intérieur de lui-même à l'aide de la création douloureuse mais salutaire pour ouvrir l'horizon de la liberté divine recouverte par les universaux culturels qui la limitent. C'est la condition sine qua non sans laquelle la liberté est inaccessible et impensable. L'homme ne peut pas s'attendre que la culture mette tout en ordre elle-même et qu'il reçoive la liberté comme un don qui n'est pas obtenu avec peine et, par conséquent, qui est injuste, comme un effet produit par l'«amélioration » des conditions de vie et la réconciliation des contradictions. La culture qui impose à l'individu ses universaux (même s'ils copient seulement les dogmes religieux) dégénère en système d'appels formels qui dévoilent leur fausseté dans tout conflit sérieux avec la vie; ils substituent et discréditent la spiritualité.

Cependant, le chemin d'une telle liberté a un haut prix pour la raison humaine. Celle-ci doit, comme les «réformateurs» le croient, se nier ellemême ayant reconnu qu'il n'y a pas d'accord entre elle et la vie humaine. Les prétentions de la raison de «régner sur le monde» sont injustifiées et destructives. Mais la raison ne peut pas refuser ces prétentions par sa propre nature. L'homme a donc besoin de la «supra-rationalité» qui consiste à refuser les prétentions irréalisables de la rationalité. Celle-ci doit céder la place à « une origine irrationnelle» qui promet la liberté. "Une origine irrationnelle se révèle dans le contenu de la vie et le mystère de la liberté est caché dans celle-là »³. C'est le christianisme qui découvre cette origine,

2. Н. А. Бердяев, Стилизованное православие (Отец Павел Флоренский) // П. А. Флоренский: pro et contra. Личность и творчество Павла Флоренского в оценке русских мыслителей и исследователей. Антология, СПб., Издательство Русского Христианского Гуманитарного Института, 2001, с. 277-278 [N. Berdiaev, «Une orthodoxie stylisée (le père Pavel Florensky) », in Pavel Florensky: pro et contra. L'individualité et la création de Pavel Florensky selon les penseurs et les chercheurs russes. Une anthologie, D. Bourlaka (dir.), Saint-Pétersbourg, Éditions de l'Institut russe chrétien des sciences humaines, 2001, p. 272-278].

3. Н. А. Бердяев, Миросозерцание Достоевского // Н. А. Бердяев о русской философии, Свердловск, Издательство Уральского университета, 1991, ч. 1, с. 6о [N. Berdiaev, "L'esprit de Dostoievski», in Nicolas Berdiaev de la philosophie russe, Sverdlovsk, Éditions de l'université de l'Oural, 1991, vol. 1, p. 60]. Traduction française: N. Berdiaev, L'esprit de Dostoïevski, Paris, Saint-Michel, 1929 (trad. par L. Julien Cain), p. 57. 
c'est sa mission: en découvrant le potentiel créatif de la liberté humaine il conduit à la foi au travers du désert de la culture où cette foi s'est éteinte.

Ainsi, les chemins de la philosophie russe religieuse se séparent. Il s'agit de changer le sens des universaux culturels, y compris la réforme de la rationalité. Mais l'essentiel de la réforme est compris d'une manière différente. La philosophie de l'omni-unité trace la voie de l'individu vers la culture, celle des «réformateurs» se retourne de la culture vers l'individu.

La pensée de Soloviev appelle la raison à se transfigurer, à s'unir avec la morale, ce qui est possible si la raison et la morale sont animées par la foi. Les « réformateurs» engagent la raison et la morale à se soumettre à la foi dans sa dimension existentielle et à lui céder la place dans la vie spirituelle de l'homme.

La raison se met également au service de la liberté et de l'esclavage. Car la liberté est fondée sur la raison qui unit l'homme avec Dieu, l'esclavage s'appuie sur la rationalité «sécularisée» qui a rompu le lien avec l'Absolu. La raison transfigurée assume une responsabilité morale, la raison sécularisée se croit un régulateur autosuffisant et même puissant sur toutes les manifestations humaines: des soucis pragmatiques et cognitifs aux valeurs morales et éthiques. Si ces deux sens sont distingués, il n'y a pas de paradoxe. S'ils sont confondus, le paradoxe de la rationalité et le paradoxe analogique de la liberté apparaissent. Mais la nécessité de distinguer ces sens ne peut être comprise qu'à l'intérieur de la culture qui, parmi ses universaux, aurait déjà celui de la rationalité animée par la foi. Dans le cas contraire, la liberté et la raison en tant qu'universaux culturels sont paradoxales, et il y a des fissures dans les fondements de la culture. Une telle culture se dégrade et meurt. Comment peut-on arrêter la dégénérescence? Il faut transformer la raison et repenser la liberté.

Ce chemin est tracé par Soloviev. Mais, à la fin du chemin, la figure de l'Antéchrist apparaît devant le regard du penseur. Cette figure incarne la «victoire» de la culture sans la foi, bien que des formes de l'organisation de la société humaine qui sont rationnelles mais vides à l'intérieur se conservent, se gonflent et atteignent l'universalité. Elles se gonflent pour éclater pendant le cataclysme dernier. La transfiguration de la raison subit un échec, la liberté est rejetée. C'est un échec de la culture et l'Apocalypse.

Avec son «sens» aigu de la métaphysique Pavel Florensky (1882-1937) avait prévu la catastrophe culturelle du $\mathrm{XX}^{\mathrm{e}}$ siècle au début de son processus et, ensuite, quand les symptômes catastrophiques furent évidents, il eut besoin de repenser la stratégie de la survie de la philosophie (et de la théologie) dans leurs nouvelles conditions spirituelles. Peut-être son « caractère 
mystérieux», son ambiguïté et son "antinomisme» font-ils peur aux uns et les en détournent et charment et attirent les autres. On peut supposer qu'au fond de son âme il y a eu l'idée de «s'habituer à la catastrophe», de la recevoir en tant que réalité, de reconnaître l'impossibilité de vivre et de penser de telle façon que la catastrophe n'est qu'un triste épisode dont le retour à l'état harmonieux de l'être s'ensuit.

Même si, comme le dit Hamlet, le monde a perdu son équilibre, il faut vivre dans ce monde détraqué en conservant la raison et la foi. Et la liberté... Qu'est-ce que signifie «être libre» sur les ruines de la culture? Libre de quoi? Pendant que la culture est solide, il est simple de spéculer si ses limites sont étroites ou larges pour une existence impatiente. On pourrait même permettre une révolte contre la culture à condition que l'on soit sûr à l'avance que celle-là sera étouffée et que l'on soit déjà d'accord d'accepter le sort de Sisyphe. Mais quand la culture est à l'agonie, cette révolte est une danse barbare sur les ruines. Et la liberté de la raison? Mais quelle raison prétend être entièrement libre et même peut s'identifier à la liberté? Quel est le rapport entre cette raison et la spiritualité?

Florensky choisit de manœuvrer entre les ruines de la culture pour trouver une source d'énergie qui alimente les forces revitalisantes. Cette source est la foi qui doit survivre après la catastrophe et mobiliser ses forces pour la surmonter. Les travaux de restauration commencent par un examen des ressources nécessaires. C’est le point de départ de la pensée de Florensky.

Tout d'abord, il reconnaît que le rationalisme européen est incapable d'être une ressource satisfaisante. Le rationalisme moderne (même sous la forme universalisée de dialectique hégélienne) n’est pas adéquat pour l'époque des catastrophes. La tentative de lui insuffler une nouvelle vie en le réunissant avec la foi dont il s'éloigne pendant deux siècles est utopique et il n'y a pas de temps pour sa vérification historique. Mais cela ne signifie point qu'il faut rejeter le rationalisme, comme le croient les «réformateurs». On a besoin de l'adapter à de nouvelles conditions. La science, par exemple, montre comment on peut le faire: ses changements provoquent la destruction des châteaux en Espagne construits selon le projet cartésien. Mais la valeur de la rationalité est conservée grâce aux mutations des problèmes scientifiques. Elle se conserve parmi les universaux culturels qui ne se détruisent pas avec la culture et maintiennent leur être idéal et autonome. Ils peuvent fournir des matériaux de restauration.

Florensky commence par le fondement. Il affirme que les lois de la logique, les vérités évidentes ou «les lois de la nature» ouvertes par la science ne se trouvent pas à la base de la rationalité. La rationalité s'appuie sur les antinomies. 
Cette doctrine de Florensky est dirigée contre Kant selon qui les antinomies témoignent que la raison n'est pas compétente dans la sphère nouménale. Elle est également dirigée contre Descartes, selon qui l'antinomie est une preuve de l'erreur dans l'application méthodique de la raison, et contre les positivistes qui essaient de construire à l'aide de la logique un fondement empirique solide pour une science délivrée de la métaphysique, de la religion et de l'idéologie. Elle est, enfin, dirigée contre Soloviev selon qui les antinomies proviennent de la destruction de l'omni-unité.

Florensky pense, d'ailleurs, que les antinomies de la raison proviennent de sa «culpabilité». Mais à la différence de Soloviev, il ne croit pas que les antinomies cachent la vérité de l'être ou qu'elles découvrent et démontrent l'impuissance de la raison comme les «réformateurs" l'imaginent. Les antinomies donnent à la raison (coupable et déchue) la possibilité de «voir » la vérité. Celle-ci est présentée sous forme d'antinomies. Sous quelle autre forme peut-elle s'ouvrir à la raison? Sous forme de jugements cohérents? Mais,

Il est clair que chaque jugement est contingent, c'est-à-dire qu'on peut lui opposer une objection sous forme d'un autre jugement et même sous celle d'un jugement contradictoire. Et si jusqu'à présent et jusqu'ici une telle objection ne s'est pas présentée, cela ne garantit nullement l'irréfutabilité de notre jugement à l'avenir ni ailleurs ${ }^{4}$.

Si le jugement peut être «annulé», il ne représente pas, bien sûr, la vérité ontologique ou divine. Cela signifie, selon Florensky, que la vérité divine ne peut point être ouverte à la raison limitée par l'exigence de non-contradiction, c'est-à-dire, à la raison soumise aux lois de la logique ordinaire.

La rationalité transfigurée doit changer le rapport à l'antinomisme. L'antinomie n'est pas un échec de la raison mais son épreuve. C'est une épreuve de sa liberté... de soi-même, c'est-à-dire, de sa forme qui est soumise aux «lois» imaginées par elle-même (on peut nommer cette forme «l'entendement» quoique l'introduction de ce terme entraîne des malentendus que nous évoquons plus loin). Après avoir traversé des épreuves, la raison peut sortir au-delà de soi-même et obtenir la foi. Dans le cas contraire, elle quitte le chemin de la foi par peur de la contradiction et, par suite, de la Vérité. Alors, la raison libérée est substituée par sa projection plane positiviste. Donc, elle ne se trouve pas au fondement de la culture où une "fissure" apparaît. Elle devient de plus en plus profonde au fur et à mesure que l'on édifie une construction colossale mais fragile au-dessus.

4. P. Florensky, La colonne et le fondement de la vérité, Lausanne, l'Âge d'homme, 1975 (trad. par C. Andronikof), p. 100 (édition citée, sauf mention particulière). 
Voilà la raison du risque pris par Florensky. Si l'antinomie est un mode d'être de la Vérité (le $V$ majuscule signifie sa nature ontologique et divine), non seulement la connaissance du monde, mais aussi la connaissance de Dieu est antinomique. La foi même considérée à travers le prisme de la rationalité est antinomique: «les antinomies sont des parties constituantes de la religion, à la considérer selon l'entendement. La thèse et l'antithèse, telles la chaîne et la trame, composent le tissu même de l'expérience religieuse. Là où il n'y a pas d'antinomie, il n'y a pas de foi $[\ldots] »^{5}$. Toute la réalité, y compris l'univers culturel, est présentée à la raison sous forme d'antinomies. Et quand le penseur dit: «la vérité est une antinomie; elle ne peut pas ne pas l'être ${ }^{6}$, il formule non seulement un principe méthodologique qui embrasse tout le domaine de la philosophie, non seulement un principe de la Weltanschauung mais un principe de la vie elle-même au sein de la culture.

L'antinomisme est, peut-être, le trait le plus connu et le plus étudié de la philosophie de Florensky. Mais, comme le souligne le philosophe russe contemporain Anatoly Akhoutine, c'est ce trait qui est fréquemment interprété d'une façon erronée ${ }^{7}$. On l'identifie à la conception kantienne des antinomies de la raison pure contre quoi proteste Florensky lui-même ${ }^{8}$. Eugène Troubetzkoy croit que Florensky confond les concepts de contradiction et d'opposition et, par conséquent, il se trompe en trouvant l'antinomisme dans les dogmes chrétiens:

L'antinomie et l'antinomisme sont, en général, enracinés dans l'entendement discursif des mystères universels; quand on va au-delà de l'entendement discursif, les antinomies sont résolues - les contradictions se transforment en coïncidence des opposés (coincidentia oppositorum) - la résolution s'accomplit au fur et à mesure de notre démarche. Voilà une réponse véritable à la question sur la résolution des antinomies en général, y compris les antinomies religieuses ${ }^{9}$.

5. Ibid., p. 111.

6. Ibid., p. 101.

7. Voir A. В. Ахутин, «София и черт: Кант перед лицом русской религиозной метафизики » // Вопросы философии, № 1, 1990, с. 63 [A. Akhoutine, «Sophia et le Diable: Kant en face de la métaphysique religieuse russe», Voprosy filosofii, no 1,1990, p. 63].

8. «Dans sa structure la plus fine, la raison est intégralement antinomique. Les antinomies de Kant ne font qu'entrouvrir la porte des coulisses de la raison » (P. Florensky, La colonne et le fondement de la vérité, p. 308).

9. Е. Н. Трубецкой, Свет Фаворский и преображение ума. По поводу книги священника П. А. Флоренского Столп и утверждение истины // П. А. Флоренский: pro et contra..., c. 297 [E. Troubetzkoy, «La lumière du Thabor et la transfiguration de la raison. À propos du livre du père Pavel Florensky, La colonne et le fondement de la vérité», in Pavel Florensky: pro et contra..., p. 297]. 
Nicolas Berdiaev, d'une part, donne une appréciation positive de l'antinomisme de Florensky («Tout ce que le père Florensky dit de l'antinomisme est excellent $\left.[\ldots] »^{10}\right)$, mais, d'autre part, il lui reproche de chercher une résolution des antinomies dans l'ontologie métaphysique alors qu'on ne peut les trouver que dans l'expérience religieuse individuelle ${ }^{11}$. La différence des interprétations de l'antinomisme chez Florensky découle de la différence des "programmes de transfiguration de la raison" qui sont évoqués au début de l'article (la philosophie de l'omni-unité et la philosophie existentielle).

Quelle est la position de Florensky lui-même? Il est difficile de la reconstruire. Dans les textes où il parle des antinomies, il y a des affirmations qui prêtent à des lectures multiples. Par conséquent, les interprétations des différents critiques divergent. Il est évident, par ailleurs, que l' «antinomie» est une clé de la philosophie de ce penseur. Le contenu de ce concept doit, donc, être expliqué au sein de toute sa philosophie et à l'aide de ses propres significations.

Ces significations sont examinées dans l'article d'Akhoutine déjà mentionné. Les antinomies de la raison pure de Kant et celles de Florensky ne sont semblables qu'en apparence; en profondeur, leurs essences internes sont contraires:

Là où Kant voit les ténèbres nouménales de la «chose en soi», la lumière de la vérité brille pour les penseurs russes. Là où la raison pure (théorique) de Kant est suspendue dans le vide sans aucun fondement, les penseurs russes découvrent un fondement absolu et supra-rationnel et «se rappellent » qu' ils s'y sont toujours trouvés. Bref, là où Kant entre dans la sphère de la philosophie même par la critique de la raison scientifique (au sens strict moderne du mot) les penseurs russes deviennent théosophes, sophiologues, théologiens. L'orientation même de la pensée se transforme, son centre est déplacé de l'intérieur de la raison autonome à l'intérieur de la Vérité elle-même. On doit la découvrir, l'expliquer, l'articuler dans le logos de la raison qui n'est pas déjà philosophique mais théosophique (désirant ardemment la sagesse) ${ }^{12}$.

Florensky oppose à la raison autocentrique ("sécularisée», séparée de la foi) de la philosophie moderne une orientation théocentrique de la raison transfigurée par la foi qui n'est pas « une faculté particulière mais un mode d'inclure d'une façon réelle l'être humain tout entier dans un plan divin du monde $»^{13}$. La raison ("pure» non parce qu'elle est «purifiée» du contenu de l'expérience mais parce qu'elle est «sophianique», touchée par

10. N. Berdiaev, «Une orthodoxie stylisée...», p. 274.

11. Ibid., p. 275.

12. A. Akhoutine, «Sophia et le Diable... », p. 64.

13. Ibid., p. 65 . 
la pureté divine) est en "procès contre elle-même» et y engage la volonté morale et la foi ${ }^{14}$. On se rappelle le point suivant: la raison moralement responsable et digne de confiance, voilà la liberté selon Soloviev. Florensky ne cherche-t-il pas cette liberté, cette «raison de la liberté» ou cette forme «sous laquelle la liberté est un fondement réel de l'“application" théorique et pratique de la raison ${ }^{15}$ ? Mais pourquoi cette forme ne peut-elle pas ne pas être une antinomie?

Si la Vérité divine (ontologique) est présentée sous l'apparence de l'antinomie est-elle antinomique elle-même? En ce cas-là, l'antinomie ne serait pas une apparence de la vérité mais la Vérité même. Mais... peut-on imaginer l'Absolu comme une antinomie? Peut-on penser l'impensable? La foi est séparée de la raison et l'on est à un pas de nommer la vérité de la raison non seulement supra-rationnelle mais aussi antirationnelle. Un autre philosophe russe contemporain, Sergey Khoroujy lit le jeune Florensky de cette manière:

Ici on voit l'envie d'accentuer toutes les contradictions propres au travail de la conscience et à la vie intérieure; la fidélité à la raison et la communion avec l'Église sont opposées et l'on affirme un irrationalisme extrême, non simplement une supra-rationalité mais une anti-rationalité des vérités de la foi. Après l'examen derrière cette opposition on peut voir une image simplifiée et, pour ainsi dire, extrémiste de la conscience qui ne peut avoir que deux formes opposées: l'entendement soumis à la logique formelle et «la raison pleine de grâce et purifiée par la prière et l'ascèse». Résultat: un logicisme étroit ou une mystique pure qui sont tout à fait hostiles ${ }^{16}$.

Pourtant, ayant fait ce dernier pas, on perd tout de suite le but pour lequel la recherche de Florensky est accomplie: il n'y a, bien sûr, aucune unité entre la raison et la liberté bien que celle-là ne soit possible que dans la foi.

L'évêque Feodor (Alexandre Pozdeevsky) «corrige» Florensky d'une manière semblable. Il croit, comme Eugène Troubetzkoy, que la confusion entre les concepts d'entendement et de raison dont Florensky se sert "sans distinction suffisante $»^{17}$ mène à une impasse. La méfiance envers

14. Ibid., p. 68

15. Ibid.

16. С. С. Хоружий, О философии священника Павла Флоренского // П. А. Флоренский, Сочинения в трех томах, t. 1, Столп и утверждение истины (I), М., Правда, 199о, c. XIV-XV [S. Khoroujy, "De la philosophie du père Pavel Florensky», in P. Florensky, Euvres en trois volumes, vol. 1, La colonne et le fondement de la vérité, Moscou, Pravda, 1990, p. XIV à XV].

17. Еп. Феодор. [Рец. на книгу], О духовной Истине. Опыт православной теодицеи (Столп и утверждение Истины). Книга свящ. П. Флоренского. М., 1912 // П. А. Флоренский: pro et contra..., c. 225 [L'évêque Feodor (compte rendu), «De la Vérité spirituelle. Essai d'une théodicée orthodoxe (La colonne et le fondement de la vérité). Livre du père Pavel Florensky, Moscou, 1912», in Pavel Florensky: pro et contra..., p. 225]. 
l'entendement si elle est exprimée par l'entendement lui-même est contradictoire. Aussi ne peut-on parler du caractère paradoxal de l'entendement que du point de vue de la raison à qui l'Absolu se révèle.

Il nous semble que l'entendement n'est pas antinomique en lui-même, mais seulement si on le compare à la vérité chrétienne de la Trinité etc., et seulement par rapport à elle; de plus, le fondement principal de les distinguer [il s'agit de l'entendement humain et de la Raison divine] est le suivant: notre entendement se fonde sur la distinction de ce qu'il distingue en le faisant la loi et la norme de l'être (d'où le besoin de discours), et la Raison divine ouverte dans le dogme reconnaît la distinction de ce que l'entendement distingue et a distingué et, en même temps, affirme et part de l'identité de ce qu'il distingue et a distingué ce qui est pour la Raison la norme de l'être supérieur et l'objet à savoir et à contempler dans la foi sans aide de la connaissance discursive ${ }^{18}$.

Autrement dit, il n'y a aucun antinomisme de la raison, les antinomies sont un lot de l'entendement que Florensky prend par erreur pour la raison.

Il faut dire que Florensky, en fait, donne des raisons pour qu'on lui reproche une distinction ambiguë entre l'entendement et la raison. Il n'est pas difficile de trouver dans ses textes (notamment dans La colonne) des passages correspondants où les deux concepts sont employés de telle façon qu'il est facile de les confondre.

Voilà l'une de ses réflexions:

[...] il est certes possible d'affirmer que la connaissance de la vérité exige la vie spirituelle et, par conséquent, qu'elle est une ascèse. Or, l'ascèse de l'entendement, c'est la foi ; autrement dit, le renoncement à soi-même. L'acte d'abnégation de l'entendement consiste précisément à exprimer une antinomie. Au demeurant, on ne peut croire qu'à une antinomie. La raison, simplement, accepte ou rejette tout jugement non antinomique, car celui-ci ne dépasse pas la frontière de l'individualité égoïste de la raison. Si la vérité était non antinomique, la raison, tournant toujours dans son propre plan, n'aurait pas de point d'appui, ne verrait pas d'objet non rationnel; par conséquent, rien ne la pousserait à commencer l'ascèse de la foi. Ce point d'appui est le dogme. C'est à partir du dogme que commence notre salut, car seul le dogme, étant antinomique, n'opprime pas notre liberté et donne libre cours soit à la foi bénévole, soit à l'incroyance maligne ${ }^{19}$.

Il est clair qu'au moment où l'antinomie devient objet de la réflexion l'entendement a déjà quitté la scène dans l'acte d'abnégation. «On peut croire

18. L'évêque Feodor, «De la Vérité spirituelle. Essai d'une théodicée orthodoxe... », p. 230.

19. P. Florensky, La colonne et le fondement de la vérité, p. 101. 
à une antinomie ", la foi entre sur la scène; maintenant la connaissance de la vérité est accomplie par ses efforts. Où est la place de la raison? Elle semble absente parmi les personnages.

La clé est dans le mot «liberté». L'entendement ayant rencontré la vérité du dogme sous forme d'antinomie se trouve devant un choix : rejeter le dogme (voir en lui une contradiction, c'est-à-dire un mensonge) ou renoncer à lui-même et se soumettre à la foi (en reconnaissant que la vérité est au-delà de l'entendement). Mais ce choix est impossible et impensable pour l'entendement. Il ne peut pas s'abandonner à la foi en restant soimême. Il ne peut pas le faire car il n'est pas libre: la liberté est incompatible avec l'entendement qui est esclave de ses propres règles et prescriptions. Autrement dit, parmi les critères qui déterminent (limitent) l'activité de l'entendement il n'y a pas de règle qui permette à l'entendement de manquer à ses propres règles, de ne pas correspondre à ces critères. En les transgressant, l'entendement reconnaît sa "folie», son «irrationalité», mais il obtient la liberté. Celle-ci est un critère véritable de la raison: la raison ne se trouve elle-même que dans la liberté.

Mais il ne s'agit pas de l'arbitraire sinon on ne pourrait pas distinguer la raison de la folie et simplement de la bêtise. Toute fuite de la prison des règles de l'entendement n'amène pas à la liberté de la raison, mais seulement celle qui est dirigée par le dogme antinomique, par la présence de la Vérité à laquelle on croit. Ainsi ce n'est pas la raison qui amène de l'entendement à la foi, c'est la foi qui dirige l'entendement vers la raison.

Maintenant on voit la réponse à la question qui met dans une impasse Eugène Troubetzkoy. Il trouve dans La colonne un conflit entre deux thèses opposées: l'antinomisme est un effet du défaut (de la "culpabilité») de l'entendement et l'antinomisme est propre à la vérité même du dogme religieux; il voit ici l'ambiguïté et l'incohérence de la pensée de Florensky (c'est un écart du chemin tracé par Soloviev et, donc, une erreur) ${ }^{20}$. Mais Eugène Troubetzkoy commet une faute: Florensky quitte le chemin de Soloviev non parce qu'il s'est trompé mais parce qu'il ouvre sa propre voie, la voie de l'interprétation symbolique des vérités de la raison.

Le dogme de la foi est antinomique. Cela signifie que la raison transfigurée par la foi voit la Vérité à travers l'antinomie. L'antinomie est un symbole de la Vérité.

Toute la philosophie de Florensky est un symbolisme si étendu et si profond qu'il embrasse toutes les sphères de sa pensée.

20. E. Troubetzkoy, «La lumière du Thabor et la transfiguration de la raison... », p. 293. 
Le concret, principe caractéristique et essentiel de la métaphysique de Florensky, ne signifie rien d'autre qu'un symbolisme, c'est-à-dire, toute la réalité est composée de symboles [...]. La réalité tout entière est symbolique, et le monde est une réunion de doubles symboles, en même temps nouménaux et phénoménaux [...]. Le symbole réunit en soi le naturel et le spirituel, de sorte que le symbolisme rejette la division de la réalité en règne des objets sensibles et des objets de l'esprit qui seraient isolés l'un de l'autre. La réalité est unie, elle est partout, dans tout son élément, aussi bien sensible que spirituelle. La structure de la connaissance est ainsi différente. Elle devient plus uniforme: dans toute sphère connaître signifie découvrir la nature symbolique des phénomènes divers, percevoir un noumène dans un phénomène; les distinctions principales entre les genres de la connaissance disparaissent donc ${ }^{21}$.

Il y a un lien essentiel entre le symbolisme et l'antinomisme: c'est dans l'antinomisme que le symbolisme trouve son expression suprême.

Le symbole accumule le contenu infini de la réalité ${ }^{22}$ et joue un rôle de «pont» entre celle-ci et la raison. Passer ce pont c'est un acte de foi car ce pas exige une abnégation (un sacrifice accompli par l'être humain fini à l'être infini pour s'unir à ce dernier) et un courage animé par la foi en ce que cette voie est sainte et unique.

L'antinomie chez Florensky n'est pas une difficulté provisoire de la connaissance ou un paradoxe qui peut être résolu à l'aide de la logique et de l'expérience. Elle réunit le monde fini et "atomisé» (désintégré par la chute) qui embrasse aussi bien l'homme que la totalité et la plénitude de l'Absolu. Elle ne sépare pas, mais réunit. Comme Sergueï Boulgakov, Florensky aussi ne voit pas dans les antinomies l'impuissance de la raison qui amène au « désespoir théorique ${ }^{23}$, mais, au contraire, l'espérance que la Vérité n'est pas inaccessible et indifférente à l'homme ${ }^{24}$. Contrairement

21. С. С. Хоружий, Философский символизм П. А. Флоренского // П. А. Флоренский: pro et contra..., c. 525-526 [S. Khoroujy, "Le symbolisme philosophique de Pavel Florensky», in Pavel Florensky: pro et contra..., p. 525-526].

22. "L'universalité du symbole contient implicite tout symbolisé bien qu'il soit infini», А. Ф. Лосев, Логика символа // Философия, мифология, культура, М., Политиздат, 1991, c. 272 [A. Losev, «La logique du symbole», in Philosophie, mythologie, culture, Moscou, Politizdat, 1991, p. 272].

23. C'est un tel désespoir qu'un chercheur russe, Yakovenko, remarque dans la conception de Florensky: «Le désespoir n'est pas ici une coloration subjective de la construction ou de l'expérience objective, mais un facteur systématique principal qui dirige tout le "travail" et qui est un noyau central de cette "confession" tout entière», Б. В. Яковенко, Философия отчаяния // П. А. Флоренский: pro et contra..., с. 255 [В. Yakovenko, «La philosophie du désespoir», in Pavel Florensky: pro et contra..., p. 255].

24. Il écrit: «Mon sort, ma raison, l'âme même de toute ma recherche, l'exigence de la certitude, je les remets entre les mains de la Vérité. Pour elle, je renonce à la preuve» (P. Florensky, La colonne et le fondement de la vérité, p. 50). 
à Boulgakov, l'antinomisme de Florensky n'a pas de tonalité tragique, il est plein d'espoir et de l'attente de la vérité, plein d'assurance dans le lien entre l'homme et l'Absolu, lien qui n'est pas rompu bien qu'il soit mis en forme d'antinomie. L'objectif de l'esprit est aussi bien de tenir ce lien comme le fil d'Ariane que d'aller dans la direction correcte ainsi que de ne pas se laisser tenter par la paix trompeuse que les images harmonieuses (et donc trop éloignées de la vérité) de l'étant promettent.

La différence entre l'antinomie et le paradoxe formel est considérable: l'antinomie dirige sa pointe vers le transcendant et conduit la pensée de la diversité apparente de l'être créé à l'omni-unité vivante; le paradoxe arrête le mouvement de la pensée en le retournant vers l'étude des causes possibles de l'erreur, si les causes sont inconnues ou irréductibles la pensée est condamnée à la stagnation ou au scepticisme. L'antinomie témoigne du mystère du transcendant au sein de l'être présent et incite à comprendre ce mystère, le paradoxe sépare l'être présent de la transcendance, il coupe le chemin de la montée de la création vers l'Absolu.

Voyons maintenant quelles sont les conséquences de l'antinomisme dans la philosophie de la culture de Florensky.

Les universaux culturels sont des «liens» qui réunissent les modes séparés de l'être humain; ils sont des formes symboliques (au sens d'Ernst Cassirer).

La philosophie des formes symboliques doit découvrir derrière les symboles et les signes une plénitude immédiate de la vie, grâce à quoi la vie même doit recevoir une forme nouvelle et authentique. La vie vient au-delà de sa présence naturelle; et on voit que sa profondeur sombre avec tout l'infini de ses manifestations extérieures s'élève vers la seule unité de son essence qui produit la totalité des manifestations vitales jusqu'aux moindres détails. «La philosophie des formes symboliques» n'est ainsi rien de plus que la philosophie de la culture ${ }^{25}$.

Toutes les manifestations de la vie sont «moulées» dans ces formes. Cependant, pour mieux comprendre le sens de ces manifestations il est nécessaire de connaître le "code», de savoir voir le contenu interne dans les «formes».

Comme tous les symboles signifiants les universaux culturels sont antinomiques. La liberté, la vérité, le bien, la beauté, enfin, la vie même

25. А. Ф. Лосев, Теория мифического мышления у Э. Кассирера // Э. Кассирер, Избранное. Опыт о человеке, М., Гардарики, 1998, с. 736 [A. Losev, «La théorie de la pensée mythologique chez Ernst Cassirer", in E. Cassirer, Euvres choisies. Essai sur l'homme, Moscou, Gargariki, 1998, p. 736]. Je crois que cette idée est tout à fait juste par rapport à la philosophie de la culture de Florensky que l'on peut également nommer une philosophie du symbole. 
sont des symboles culturaux qui embrassent les contradictions entre le fini et l'infini, l'individuel et le total.

Si les universaux de la culture sont déchirés de leur fondement spirituel (selon Florensky, de la foi) et réduits aux règles juridiques ou traditionnelles de l'être, la culture elle-même n'est qu'un ensemble de règles formelles dont le respect n'est soutenu que d'une façon artificielle. Une telle culture est vouée à la dégradation, son influence réunissante sur la vie s'affaiblit au fur et à mesure que les tendances au schisme deviennent de plus en plus fortes, les forces centrifuges nourries par l'égoïsme et les intérêts privés et séparatistes augmentent. Sa décadence est inévitable parce qu'elle «parodie» la foi.

Elle [la Culture] s'accroche avec peur aux formes de la vie religieuse et, car elle ne sait pas voir en celles-ci la présence cristallisée de l'Esprit et de la Vérité, elle les prend comme les normes d'une législation juridique. Elle les traite d'une façon extérieure et ne les tient pas comme des fenêtres qui donnent la lumière du Christ mais comme des exigences conditionnelles de l'autorité extérieure. La conscience chrétienne sait que les prescriptions de l'Église ne sont pas fortuites et sont proposées par l'Église comme une condition favorable du salut; c'est une règle et une prescription de la vie spirituelle saine de la société chrétienne. Mais il est toujours clair pour la conscience chrétienne que les prescriptions d'une hygiène spirituelle peuvent être remplacées par d'autres dans certaines conditions: le respect des exigences de l'Église par leur essence interne - de contribuer au salut - amène parfois au non-respect de la lettre ainsi que le respect de la lettre peut, également, contredire leur essence spirituelle ${ }^{26}$.

En ce sens, comme le croit Florensky, la philosophie de la culture (ainsi que la philosophie tout entière) se trouve devant un choix: soit le christianisme qui élève l'antinomisme de la connaissance vers la foi purifiante et pleine de grâce, soit une «demi-foi » qui n'affirme le statut des universaux culturels qu'en apparence et le réduit, en fait, aux contradictions formelles (parmi lesquelles il y a une contradiction entre la liberté individuelle, d'un côté, et le cadre moral et juridique de sa conduite, de l'autre), et, donc, amène à la dévalorisation de la culture.

La foi sauve la culture, la «demi-foi » qui est plus dangereuse que l'incroyance la tue. Vider l'antinomie, la réduire au paradoxe où les parties de l'antinomie sont dissociées et, ensuite, confrontées comme des oppositions abstraites signifie détruire la culture et la rendre absurde.

26. П. А. Флоренский, Записка о христианстве и культуре // Сочинения в четырех томах, М., Мысль, t. 2, 1996, с. 555 [P. Florensky, «Note sur le christianisme et la culture», in Euvres en quatre volumes, Moscou, Mysle, vol. 2, 1996, p. 555]. 
Dans une telle culture il ne reste qu'une enveloppe de la foi. Par exemple, les principes moraux peuvent conserver une forme héritée de la religion, mais ils sont privés de leur valeur religieuse et ontologique; la religion ellemême est réduite à la doctrine morale. Une telle morale et une telle religion deviennent "pharisaïques", c'est-à-dire formelles et, donc, ébranlables sous l'influence des circonstances historiques ou des passions individuelles.

Le symbolisme, en revanche, élève la culture, lui donne une possibilité d'être en rapport avec l'Absolu. La culture est un espace où le "monde terrestre» et le «monde céleste» sont croisés, c'est une frontière entre la réalité transcendante et la réalité mondaine. Les universaux culturels sont des symboles qui découvrent l'infini de la vérité, mais la représente de telle façon qu'elle ne soit pas réduite au fini, au changeant, à ce qui passe.

La culture est une valeur supérieure de l'être terrestre, elle transfigure l'homme. En ce sens elle est proche du culte. Sa nature symbolique permet à l'homme de devenir "plus qu'il est», de ne pas exister dans le monde, mais de créer l'univers ${ }^{27}$. La culture est une condition et un facteur réel de création. Ainsi Florensky s'oppose aux panégyriques anarchiques de la liberté créative de l'esprit individuel dont Nicolas Berdiaev est familier. La possibilité et la nécessité de la création se trouvent dans la nature antinomique des symboles culturaux: d'une part, ils ouvrent une perspective de transformations infinies de ces sens, d'autre part, ils mettent cette perspective en série convergente dirigée dans son mouvement vers la vérité. Ils réunissent l'enracinement ontologique et l'ouverture gnoséologique. Au sens figuré les symboles-antinomies culturels sont des voies de liaison entre l'homme et la Transcendance. La foi soutient ces voies en marche, elle interdit de les engorger par les déchets quotidiens, de les transformer en formes sans contenu.

Aussi, la métaphysique antinomique et symbolique de la culture estelle liée, d'un côté, à la théologie et, de l'autre côté, à la "physique» de la culture (aux sciences de la culture), en se nourrissant en même temps à ces deux sources. La théologie communique à la philosophie de la culture un sens ontologique, les sciences de la culture lui donnent la matière où ce sens trouve son contenu. Tout phénomène de la culture réunit l'action des universaux culturels et la «chosité»: c'est le sens d'une «métaphysique concrète» que Florensky projette et accomplit. Cela signifie que tout phénomène de la culture est un «symbole concret» qui réunit l'infini des sens et leur actualisation finie, ce qui est une antinomie.

27. «La foi détermine le culte, le culte à son tour détermine la conception du monde d'où vient la culture», П. А. Флоренский [Автореферат] // Сочинения в четырех томах, t. 1, 1994, c. 39 [P. Florensky, «Résumé de thèse», in CEuvres en quatre volumes, vol. 1, 1994, p. 39]. 
La division de la culture en sphère «matérielle» et «spirituelle» n'a qu'un caractère conditionnel, secondaire et heuristique. La culture est spirituelle par son essence. L'esprit des universaux culturels reste présent dans tout événement culturel, dans tout contenu concret et matériel de la vie humaine. On ne doit pas apprécier l'état de la «culture spirituelle» seulement en fonction de la quantité ou de la qualité des valeurs dites «culturelles» accumulées ou créées au cours de l'histoire culturelle. La culture spirituelle est un univers tout entier de la vie humaine pris en sa signification symbolique. C'est pourquoi la décadence de la culture commence au moment où le lien entre le contenu des phénomènes et leur sens symbolique est rompu, où ces phénomènes cessent d'être des symboles ${ }^{28}$.

Par exemple, le rapport de Florensky à la peinture européenne moderne est lié à cette idée : il croit qu' elle réfléchit à la corruption de la «stabilité religieuse de la Weltanschauung», que «la métaphysique sacrée de la conscience populaire commune» est remplacée par « une manière individuelle de voir un visage isolé avec son point de vue particulier, et, en outre, avec le point de vue particulier notamment à un moment donné» ${ }^{29}$. L'illusion de l'espace profond est une valeur de la conscience individualisée et «isolée » pour qui une «apparence vraisemblable» est plus importante que le symbolisme métaphysique avec sa totalité ouverte par la vue spirituelle.

L'essence de la culture est ainsi réfléchie dans ses phénomènes. C'est elle qui est au centre de l'attention de la philosophie de la culture qui, selon Florensky, cherche des conditions sous lesquelles le lien entre le contenu réel et symbolique est conservé ou perdu. La philosophie de la culture est une «thérapie» qui examine les causes des maladies de la culture et donne les moyens pour les guérir. Les graves crises et les catastrophes de la culture, en partie, ont lieu parce que la philosophie de la culture manque cette fonction et dégénère en "comparatisme» qui s'occupe d'une façon abstraite de la typologie, de la classification, de la description des événements et des faits culturels, c'est-à-dire qu'elle devient une «science de la culture».

28. Florensky attache une signification exceptionnelle aux formes et aux procédés de l'art qui permettent de sentir et de comprendre le symbolisme de l'Univers. Par exemple, il parle de la perspective inversée dans la peinture d'icônes avec son symbolisme spatial, des aspects artistiques de la liturgie qui lie le concret de l'être humain avec la totalité du contenu spirituel, etc. Ces phénomènes sont tout à fait différents par rapport aux recherches formalistes (bien qu'ils reproduisent certains procédés de l'art médiéval): la différence principale des Weltanschauungen se trouve derrière une ressemblance apparente.

29. П. А. Флоренский, У водоразделов мысли (Черты конкретной метафизики) // Сочинения в четырех томах, М., Мысль, t. 3(1), 200о, с. 52 [P. Florensky, «La ligne de partage de la pensée (Traits de la métaphysique concrète) ", in CEuvres en quatre volumes, Moscou, Mysle, vol. 3(1), 200o, p. 52]. 
Si la culture est un système de symboles universaux antinomiques, la multiplicité des cultures découle du fait que de tels systèmes peuvent différer aussi bien par les composants que par la structure. Ils ne se partagent pas en plus ou moins "progressifs» (on ne peut pas représenter les cultures comme des étapes d'une "évolution" $)^{30}$. Néanmoins, il existe une unité entre les cultures différentes (leurs différences et leurs ressemblances doivent occuper la typologie des cultures) : elles sont des moyens pour découvrir et mettre en œuvre les valeurs absolues qui sont des objets de la foi ${ }^{31}$.

Est-ce que Florensky reconnaît l'équivalence culturelle des cultes différents, c'est-à-dire, est-ce qu'il voit dans tout culte une conception symbolique de la Transcendance unique, conception qui est particulière, mais égale à d'autres par rapport à la vérité? Certes, non.

Il souligne, par exemple, que la culture de la Renaissance européenne se caractérise par «la séparation, la subjectivité, l'abstraction et la superficialité ${ }^{32}$. Ce sont des caractéristiques négatives, contraires aux appréciations positives de la culture «de type médiéval». Mais peut-on, en fait, donner les appréciations "positives» ou "négatives» sur les cultures si l'on reste sur le terrain du symbolisme culturel?

Florensky dit que la vie des cultures particulières est soumise à un certain rythme dans lequel le type «médiéval» et le type «renaissant» dominent tour à tour mais sous formes différentes et spécifiques. Par exemple, à son avis, au début de $\mathrm{XX}^{\mathrm{e}}$ siècle la culture européenne commence à se manifester dans toutes les sphères comme « un nouveau Moyen Âge» qui va remplacer la renaissance européenne qui termine son existence. Puisque Florensky lui-même reconnaît que sa Wetlanschauung est proche au type médiéval, on pourrait conclure qu'il salue les changements culturels qui entourent sa vie à la fin du premier quart du siècle précédent. Mais cette interprétation contredirait ce qu'on a dit au début de cette étude sur ses intuitions et ses sentiments par rapport à la catastrophe culturelle. Mais la fixation de cette contradiction serait naïve.

La culture, d'après Florensky, est hétérogène, elle a des couches, des strates, des niveaux différents. Ce sont des niveaux du symbolisme et de sa perception, c'est-à-dire, de la capacité de comprendre le symbolisme, de voir le mystère de l'univers et son Sens à travers celui-ci. Ce sont des niveaux d'une pratique particulière qui consiste à symboliser le monde, à décrire les symboles et à faire leur typologie, à créer et à recréer les conditions sous lesquelles les symboles fonctionnent notamment en tant que symboles et

\footnotetext{
30. Voir P. Florensky, «Résumé de thèse», p. 38

31. Ibid., p. 39.

32. Ibid.
} 
non comme des signes vides ou incompréhensibles. Au niveau inférieur du symbolisme culturel l'homme n'a pas d'accès au symbolisme le plus élevé, plus proche du culte. Mais à l'époque où le niveau culturel commun baisse (la pratique symbolique devient primitive) la couche culturelle dans laquelle la pratique du symbolisme est soutenue au niveau suprême ésotérique se conserve. Ceux qui appartiennent à cette couche sont les gardiens et les créateurs de la culture «supérieure», des prêtres du culte qui ont une "connaissance ésotérique». Ils sont destinés à dépasser la crise et à faire sortir la culture de la catastrophe. Il semble que Florensky se rattache lui-même à ce groupe.

Le négativisme de Florensky à l'égard de la culture de la Renaissance est adressé notamment aux niveaux supérieurs de son symbolisme, à ce qui constitue, pour ainsi dire, le «code» qui détermine son type. Est-ce que cela signifie que les sens de la Vérité ne sont pas vus à travers les symboles des universaux culturels de la Renaissance, c'est-à-dire, que ces symboles ne sont que des «antinomies vides», des paradoxes formels?

En ce cas-là, tous les cultes ne sont pas égaux en ce qui concerne leur signification symbolique. C'est-à-dire, tous les cultes ne produisent pas les niveaux supérieurs de la culture. Les cultures ne sont pas équivalentes, elles ne réunissent pas de la même façon le monde humain avec le monde de la Vérité. Mais l'alternance des époques culturelles (ou la coexistence des cultures différentes au même moment historique) n'est pas une évolution du bas vers le haut qu'on pourrait représenter, par exemple, comme une fonction continue, mais un rythme intermittent: une culture cède la place à une autre, peut-être plus basse pour revenir le moment venu. L'histoire de la culture est la difficile respiration de l'esprit.

Vladimir Porus Université d'État, École des hautes études en sciences économiques (Moscou) 